\title{
Late clinical outcomes of aortic valve replacement with Carpentier-Edwards pericardial valves
}

\author{
Hyoung Woo Chang ${ }^{1 \#}$, Wook Sung Kim", Joong Hyun $\mathrm{Ahn}^{3}$, Keumhee C. Carriere ${ }^{3,4}$, Dong Seop Jeong ${ }^{2}$, \\ Yang Hyun $\mathrm{Cho}^{2}$, Kiick Sung ${ }^{2}$, Pyo Won Park ${ }^{2}$ \\ ${ }^{1}$ Department of Thoracic and Cardiovascular Surgery, Seoul National University Bundang Hospital, Gyeonggi-do, Republic of Korea; ${ }^{2}$ Department \\ of Thoracic and Cardiovascular Surgery, ${ }^{3}$ Bioinformatics Center, Samsung Medical Center, Sungkyunkwan University School of Medicine, Seoul, \\ Republic of Korea; ${ }^{4}$ Department of Mathematical and Statistical Sciences, University of Alberta, Edmonton, AB, Canada \\ Contributions: (I) Conception and design: PW Park, HW Chang; (II) Administrative support: PW Park, WS Kim; (III) Provision of study materials \\ or patients: PW Park, HW Chang, WS Kim; (IV) Collection and assembly of data: PW Park, HW Chang; (V) Data Analysis and interpretation: HW \\ Chang, PW Park, WS Kim; (VI) Manuscript writing: All authors; (VII) Final approval of manuscript: All authors. \\ \#These authors contributed equally to this work. \\ Correspondence to: Pyo Won Park, MD, PhD. Department of Thoracic and Cardiovascular Surgery, Samsung Medical Center, 81 Irwon-ro, Gangnam- \\ gu, Seoul 06351, Republic of Korea. Email: pyowonpark@gmail.com.
}

\begin{abstract}
Background: The present study aimed to compare the long-term clinical and hemodynamic outcomes of aortic valve replacement using Carpentier-Edwards Perimount (Perimount) or Perimount Magna (Magna) valves.

Methods: We enrolled 430 patients who underwent aortic valve replacements with Perimount $(\mathrm{n}=58)$ or Magna ( $\mathrm{n}=372)$ valves [1998-2013]. Multivariable and inverse probability of treatment weight (IPTW) analyses were performed.

Results: Before IPTW analysis, the overall 8-year survival rate differed significantly between the groups [Perimount $90 \% \pm 4 \%$ vs. Magna 76\% $4 \%$; P=0.02; hazard ratio (HR): 0.37 for the Perimount group; $95 \%$ confidence interval (CI): 0.17-0.83]. Multivariable analysis of the overall survival identified Perimount valve use as a protective factor ( $\mathrm{P}=0.009$; HR: $0.32 ; 95 \%$ CI: $0.14-0.75)$. Independent risk factors of overall survival were older age, male sex, higher preoperative left ventricular mass index, lower ejection fraction, lower aortic valve pressure gradient, and lower haemoglobin. After applying IPTW, overall survival was again found to be significantly longer in the Perimount group ( $\mathrm{P}=0.04$; HR: 0.43; 95\% CI: 0.20-0.93). Event-free survival was also better in the Perimount group ( $\mathrm{P}=0.006$; HR: 0.38; 95\% CI: 0.19-0.75). However, the Magna group had significantly lower aortic valve pressure gradients at one year and five years postoperative.

Conclusions: Although Magna use led to decreased aortic valve pressure gradients at follow-up, overall and event-free survival rates were significantly better with use of the Perimount valve. Additional and larger studies are needed to confirm these results.
\end{abstract}

Keywords: Prosthesis; aortic stenosis (AS); aortic valve replacement; hemodynamics; survival

Submitted Sep 11, 2019. Accepted for publication Oct 31, 2019.

doi: $10.21037 /$ jtd.2019.11.65

View this article at: http://dx.doi.org/10.21037/jtd.2019.11.65

\section{Introduction}

Since its introduction in 1982, the Perimount (Perimount) pericardial aortic valve has been used widely in patients. However, the hemodynamic disadvantages of this valve are a concern, especially in patients with a small aortic annulus. The Perimount Magna (Magna) valve, introduced in 2003, reportedly overcame the hemodynamic disadvantages of the Perimount valve. The Magna valve was designed for placement in the supra-annular position 


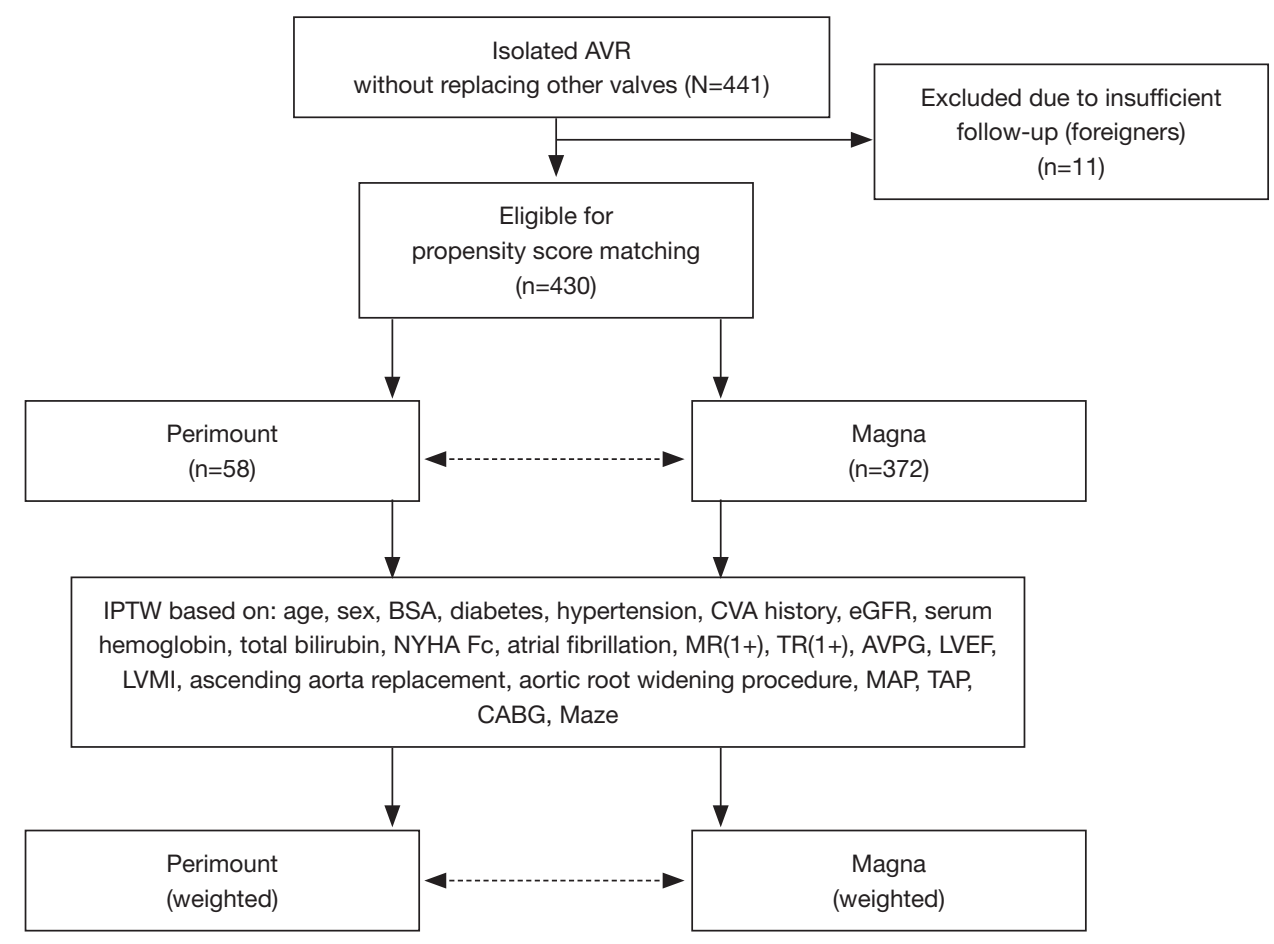

Figure 1 Study design and data analysis flow diagram. AVPG, aortic valve pressure gradient; AVR, aortic valve replacement; BSA, body surface area; CABG, coronary artery bypass grafting; CVA, cerebrovascular accident; eGFR, estimated glomerular filtration rate; IPTW, inverse probability of treatment weight; LVEF, left ventricular ejection fraction; LVMI, left ventricular mass index; MAP, mitral annuloplasty; MR, mitral regurgitation; NYHA Fc, New York Heart Association functional class; TAP, tricuspid annuloplasty; TR, tricuspid regurgitation.

and provided a larger effective orifice area (EOA) than a size-matched Perimount valve (1). This is thought to lower the transvalvular pressure gradient, a significant surgical parameter, after aortic valve replacement (AVR). However, few studies have compared the clinical use of the Perimount and Magna valve in terms of postoperative outcomes. Given this, the proposed hemodynamic superiority of the Magna valve to the Perimount valve remains untested and unproven. Few papers have compared hemodynamic parameters in the early postoperative period. Here, we compared the postoperative clinical outcomes of Perimount and Magna valve recipients with severe aortic stenosis (AS) to evaluate the hemodynamic benefits of each valve type and their effects on long-term patient survival.

\section{Methods}

\section{Study design}

This is a retrospective observational study. Data were collected through a review of electronic medical records. The hospital institutional review board of Samsung Medical Center approved this study and waived any need for informed consent due to its retrospective nature (no. 201801-013). An analysis flow diagram is shown in Figure 1. A total of 441 AVR procedures were performed for the treatment of severe AS using the Perimount or Magna valves between 1998 and 2013 at Samsung Medical Center. Cases with concurrent mitral or tricuspid annuloplasty were included; those in which any other valves were replaced in the same operation were excluded. Concomitant coronary artery bypass grafting, ascending aorta procedure, Maze procedure, and aortic root widening procedure cases were included.

\section{Surgical techniques}

All operations were performed with a full median sternotomy approach. Aortic cannulae were placed at the lesser curvature of the aortic arch and a standard 
A

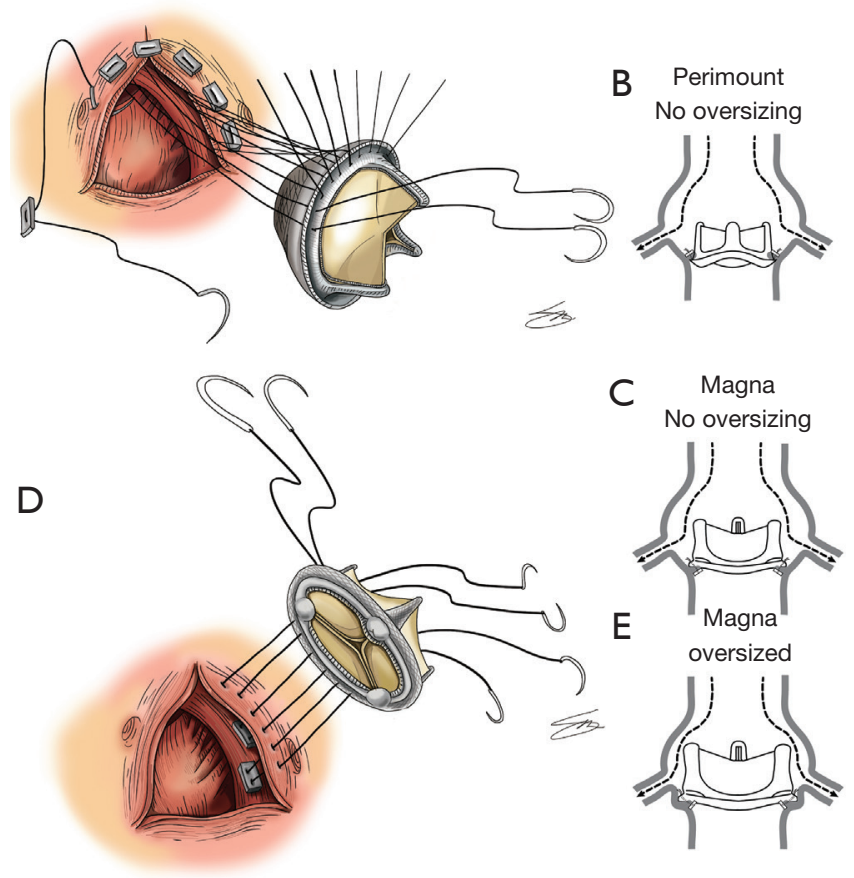

Figure 2 Surgical techniques of aortic valve implantation. (A,B) Depict intra-annular positioning of Perimount valve using an everting suture; $(\mathrm{C}, \mathrm{D}, \mathrm{E})$ depict supra-annular positioning of Magna valve using a non-everting suture; (E) depicts the distorted aortic root and the relationship between the sewing ring and coronary orifices.

bicaval cannulation was performed. Cold antegrade and/ or retrograde blood cardioplegia was used for myocardial protection. In the Perimount group, transannular everting sutures were used in most cases (Figure $2 A, B$ ). In contrast, only the supra-annular non-everting suture technique was used in the Magna group (Figure $2 C, D, E$ ). For patients with an extremely small aortic annulus, an aortic root widening procedure was performed. Ascending aorta wrapping or replacement was performed when the ascending aorta diameter exceeded $45 \mathrm{~mm}$. Patient-prosthesis mismatch (PPM) was defined as an EOA index $\leq 0.85 \mathrm{~cm}^{2} / \mathrm{m}^{2}$. The EOA index was calculated based on manufacturer-provided EOA values.

\section{Perioperative management and follow-up}

Transthoracic echocardiography was performed before discharge in all patients. In an outpatient clinic, patients were advised to undergo echocardiography at least once within the first postoperative year and then whenever possible during years two-three, three-five, and then after five years. All patients were prescribed warfarin for three months postoperatively. The target prothrombin time international normalized ratio was $1.5-2.0$. Morbidity and mortality were defined per the "Guidelines for reporting mortality and morbidity after cardiac valve interventions" (2). "Events" in event-free survival included reoperation due to structural valvular deterioration, non-structural valvular dysfunction, major bleeding requiring the transfusion of $\geq 2$ units of packed red blood cells, valve-related thromboembolism (including stroke), and prosthetic valve endocarditis.

\section{Statistical analyses}

A summary diagram of the analytic process used is presented in Figure 1. SAS 9.3 (SAS Institute, NC, USA) and PASW 20 (IBM SPSS Inc., Armonk, NY, USA) were used for all analyses. Student's $t$ and chi-square tests were used to compare continuous and categorical variables, respectively. Student's $t$-test could suffice because there were only two study groups. Fisher's exact tests were used when the categorical data were sparse. With unweighted original data, Kaplan-Meier survival curves were drawn and a Cox regression was used. Multivariable Cox analyses of overall mortality were performed to adjust the hazard ratio of Perimount use over Magna for various confounding variables. Variables that had an incidence of at least 5 were included in the multivariable analyses with the stepwise forward and backward selection method (entry probability of 0.20 , removal probability of 0.05 ), while the factor "Use of the Perimount valve" was included in the multivariable analysis, regardless of variable selection process. These clinical and demographic variables included sex, age, hypertension, serum haemoglobin level, total bilirubin, New York Heart Association functional class III or IV, EuroScore, atrial fibrillation, preoperative mean aortic valve pressure gradient, preoperative left ventricular (LV) ejection fraction, preoperative LV mass index, concomitant ascending aorta wrapping or replacement, tricuspid annuloplasty, coronary artery bypass, and the Maze procedure. The proportional hazard assumption of the Cox model was verified using Schoenfeld residuals.

Alternatively, to balance the patients for differences in baseline characteristics between the Perimount and Magna groups, an inverse probability of treatment weight (IPTW) procedure was applied. IPTW method was chosen because 
Table 1 Patient characteristics before and after inverse probability of treatment weighting (IPTW)

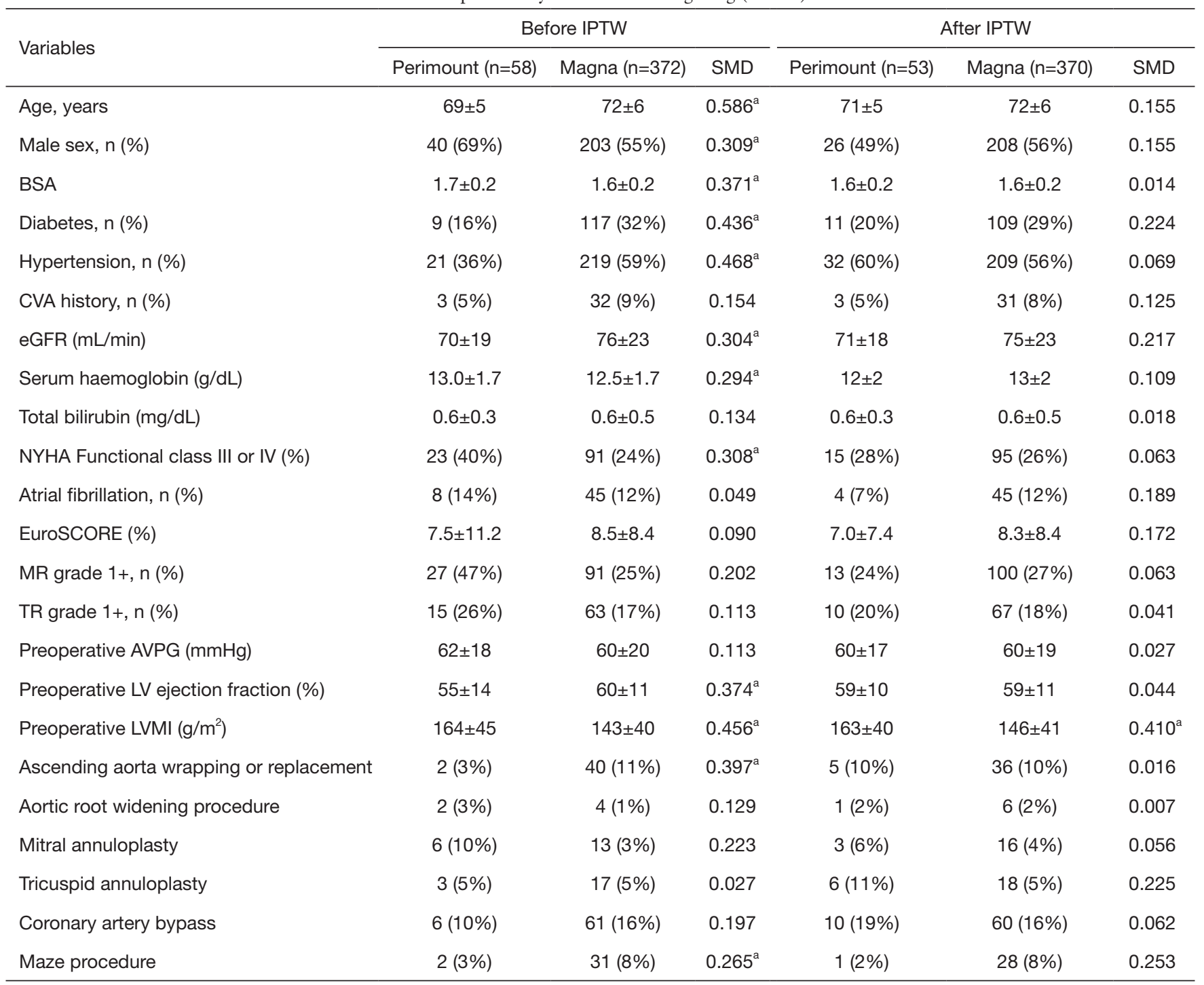

a, SMD absolute value exceeding 0.25. AVPG, aortic valve pressure gradient; BSA, body surface area; CVA, cerebrovascular accident; eGFR, estimated glomerular filtration rate; LV, left ventricle; LVMI, left ventricular mass index; MR, mitral regurgitation; NYHA, New York Heart Association; SMD, standardized mean difference; TR, tricuspid regurgitation.

it could minimize the dropouts. A logistic regression model was constructed to calculate the propensity score for each case in the Perimount and Magna groups (Table 1). After applying the IPTW procedure, acceptable standardized mean differences $(<0.25)$ were observed for all characteristics except LV mass index (Figure 3). A clustered Cox regression analysis of overall survival and event-free survival based on the IPTW procedure was performed to evaluate and reconfirm the effect of valve choice. Preoperative LV mass was included as a covariate (because it was not well balanced between the groups after applying the IPTW, as per doubly robust procedure). The difference in changes in aortic valve mean pressure gradient (AVPG) over time was assessed using a generalized estimating equation method with a Bonferroni correction for multiple comparisons. 


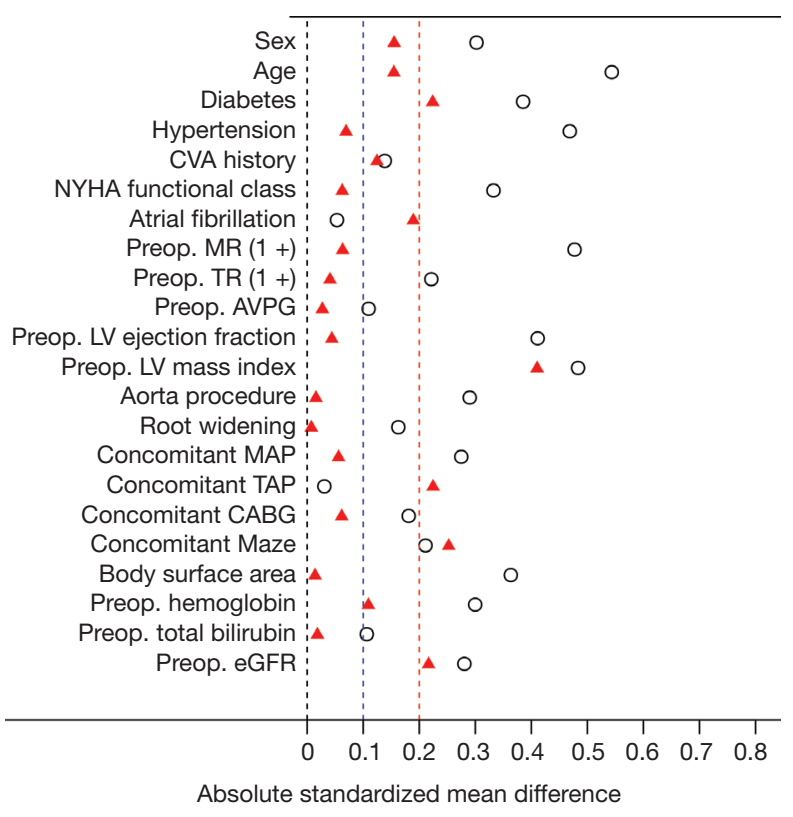

Figure 3 Patient characteristics by group. Absolute mean differences before (hollow circles) and after (red-filled triangles) inverse probability of treatment weighting. AVPG, aortic valve mean pressure gradient; CABG, coronary artery bypass grafting; CVA, cerebrovascular accident; eGFR, estimated glomerular filtration rate; LV, left ventricular; MAP, mitral annuloplasty; MR, mitral regurgitation; NYHA, New York Heart Association; Preop., preoperative; TAP, tricuspid annuloplasty; TR, tricuspid regurgitation.

\section{Results}

\section{Patient characteristics and surgical data}

Baseline patient characteristics are presented in Table 1. The mean age of the 430 patients included was $72 \pm 6$ years. Of these patients, 243 (57\%) were male. Concomitant ascending aorta replacement was performed in 43 patients $(10 \%)$. The mean preoperative aortic valve pressure gradient was $60 \pm 19 \mathrm{mmHg}$ and the $\mathrm{LV}$ mass index was $146 \pm 41 \mathrm{~g} / \mathrm{m}^{2}$. Patients in the Magna group were significantly older and more frequently had diabetes and hypertension. However, there were significantly more patients with a New York Heart Association functional class rating of III or IV in the Perimount group. LV mass index was also significantly higher in the Perimount group. PPM (EOA index $\leq 0.85 \mathrm{~cm}^{2} / \mathrm{m}^{2}$ ) calculated based on the manufacturer-provided reference EOA was significantly more common in the Perimount group. The distribution of concomitant operations did not differ significantly between the groups. There were three 30-day mortality in Magna group, but none in Perimount group $(\mathrm{P}>0.999)$. The median follow-up period was 132 [interquartile range (IQR), 122-153] months in the Perimount group and 41 (IQR, 22-69) months in the Magna group $(\mathrm{P}<0.001)$. The completeness of follow-up was $97.5 \%$ for overall mortality because survival data from national medical insurance databases was used for all patients. However, the rate of loss to outpatient clinic during follow-up was $16 \%$.

\section{Multivariable analyses of overall survival}

The results of univariable and multivariable analyses for overall mortality are shown in Table 2. Independent risk factors of overall survival were older age, male sex, higher preoperative left ventricular mass index, lower ejection fraction, lower aortic valve pressure gradient, and lower haemoglobin. Perimount valve use was a protective factor compared to Magna valve use $[\mathrm{P}=0.009$; hazard ratio (HR): 0.32; $95 \%$ confidence interval (CI): $0.14-0.75$ ], adjusted by all the significant risk factors above.

\section{IPTW applied analyses}

The propensity score adjusted balance of standardized mean differences via IPTW is shown in Table 1 and Figure 3. Before applying the IPTW procedure, the incidence of PPM was significantly higher in the Perimount group $[36 / 58(62 \%)$ in the Perimount group vs. 23/372 (6\%) in the Magna group; $\mathrm{P}<0.001]$. Higher incidence of $\mathrm{PPM}$ in the Perimount group persisted after applying ITPW [30/53 (57\%) in the Perimount group versus 22/370 (6\%) in the Magna group; $\mathrm{P}<0.001]$. Valve-related adverse outcomes adjusted by IPTW were not different between the two valve groups (Table 3).

Before applying the IPTW procedure (Figure 4), the eight-year overall survival was $90 \% \pm 4 \%$ and $76 \% \pm 4 \%$ in the Perimount and Magna groups, respectively (Perimount group: HR, 0.37; 95\% CI: 0.17-0.83; P=0.016). Eventfree survival in the Perimount group was significantly better than in the Magna group $(86 \% \pm 5 \%$ vs. $66 \% \pm 5 \%$, respectively; $\mathrm{P}=0.004$; Perimount group: $\mathrm{HR}, 0.36$; $95 \% \mathrm{CI}$ : 0.18-0.72). Survival analyses adjusted via IPTW are shown in Figure 4B,D. The Perimount group's superior overall and event-free survival rates persisted after applying the IPTW procedure. Clustered Cox regressions revealed that the Perimount group had better overall survival (HR, 0.43; $95 \%$ CI: $0.20-0.93 ; \mathrm{P}=0.031$ ). Event-free survival also indicated 
Table 2 Multivariable Cox regression analysis of overall survival using original raw data (unweighted). A stepwise variable selection approach was used

\begin{tabular}{|c|c|c|c|c|}
\hline Variables & \multicolumn{2}{|c|}{ Univariable } & \multicolumn{2}{|c|}{ Multivariable } \\
\hline Being female & $0.69(0.41-1.17)$ & 0.17 & $0.49(0.27-0.88)$ & 0.018 \\
\hline Age & $1.08(1.03-1.13)$ & 0.001 & $1.06(1.02-1.11)$ & 0.009 \\
\hline Diabetes & $1.40(0.82-2.38)$ & 0.21 & & \\
\hline CVA history, n (\%) & $0.71(0.22-2.28)$ & 0.57 & & \\
\hline eGFR (mL/min) & $1.00(0.98-1.01)$ & 0.52 & & \\
\hline Serum haemoglobin (g/dL) & $0.80(0.69-0.91)$ & 0.001 & $0.78(0.66-0.92)$ & 0.003 \\
\hline Total bilirubin (mg/dL) & $1.22(0.91-1.64)$ & 0.18 & & \\
\hline Atrial fibrillation & $1.63(0.89-3.01)$ & 0.12 & & \\
\hline MR grade $1+$ & $1.31(0.80-2.17)$ & 0.29 & & \\
\hline TR grade $1+$ & $1.38(0.80-2.38)$ & 0.25 & & \\
\hline Preoperative AVPG & $0.98(0.97-1.00)$ & 0.04 & $0.98(0.97-1.00)$ & 0.023 \\
\hline Preoperative LV ejection fraction & $0.98(0.96-1.00)$ & 0.02 & $0.98(0.96-1.00)$ & 0.048 \\
\hline Preoperative LVMI & $1.01(1.00-1.01)$ & 0.006 & $1.01(1.00-1.01)$ & 0.031 \\
\hline Use of Perimount valve & $0.37(0.17-0.83)$ & 0.02 & $0.32(0.14-0.75)$ & 0.009 \\
\hline Ascending aorta wrapping or replacement & $2.03(0.91-4.52)$ & 0.08 & & \\
\hline
\end{tabular}

AVPG, aortic valve mean pressure gradient; CVA, cerebrovascular accident; eGFR, estimated glomerular filtration rate; LV, left ventricle; LVMI, left ventricular mass index; MR, mitral regurgitation; NYHA, New York Heart Association; TR, tricuspid regurgitation; $H R$, hazard ratio.

Table 3 Valve-related complications in all patients, before and after inverse probability of treatment weighting (IPTW)

\begin{tabular}{|c|c|c|c|c|}
\hline Adverse outcomes & \multicolumn{2}{|c|}{ Before IPTW, n (\%) } & \multicolumn{2}{|c|}{ After IPTW, n (\%) } \\
\hline Paravalvular leak & 0 & $1(0.3)$ & 0 & $0.9(0.2)$ \\
\hline Prosthetic valve endocarditis & $1(1.7)$ & $3(0.8)$ & $0.2(0.4)$ & $2.8(0.8)$ \\
\hline Pannus formation & $1(1.7)$ & 0 & $0.3(0.6)$ & 0 \\
\hline $\begin{array}{l}\text { Permanent pacemaker } \\
\text { implantation }\end{array}$ & $1(1.7)$ & $5(1.3)$ & $0.6(1.1)$ & $5.0(1.4)$ \\
\hline Reoperation & $1(1.7)$ & $5(1.3)$ & $0.6(1.1)$ & $5.0(1.4)$ \\
\hline
\end{tabular}

${ }^{*}$, haemorrhage requiring $\geq 2$ packed red blood cell transfusions. 

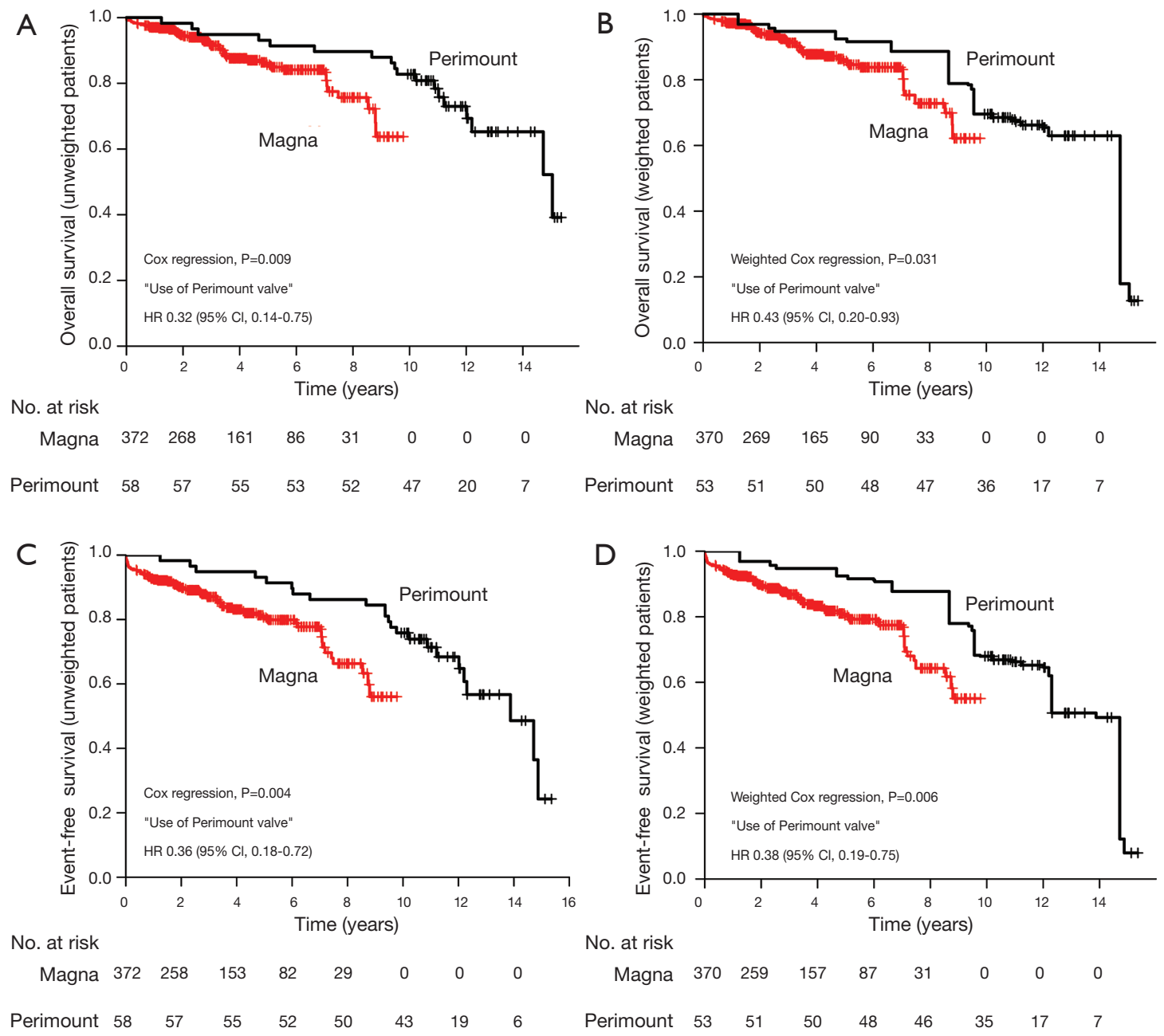

Figure 4 Survival analyses (Kaplan-Meier curves and multivariable Cox regression) before and after inverse probability of treatment weighting (IPTW). (A) Overall survival (before IPTW); (B) overall survival (after IPTW); (C) event-free survival (before IPTW); and (D) event-free survival (after IPTW). CI, confidence interval; HR, hazard ratio; No., number.

that Perimount valve use was associated with decreased risk (HR, 0.38; 95\% CI, 0.19-0.75; $\mathrm{P}=0.006$ ). We also compared long-term cardiac related mortality, and the survival curves are presented in the supplementary material. There was no significant difference in cardiac related mortality before and after IPTW (Figure S1). However, cause of death during long-term follow-up was not sufficiently reliable.

Figure 5 shows changes in AVPGs over time. The Perimount group generally had higher AVPGs, significant except at two-three and three-five years postoperative, per echocardiography.

\section{Discussion}

For the present study, we hypothesized that there would be a difference in long-term clinical outcomes between AVR cases in which Perimount or Magna valves were used. Multivariable analyses and IPTW-transformed analyses were performed to overcome differences in baseline patient characteristics between the groups. As a result, the Perimount group had significantly better outcomes than the Magna group in terms of overall and event-free survival. In contrast, AVPG was lower in the Magna group, and significant except at two-three and three-five years 


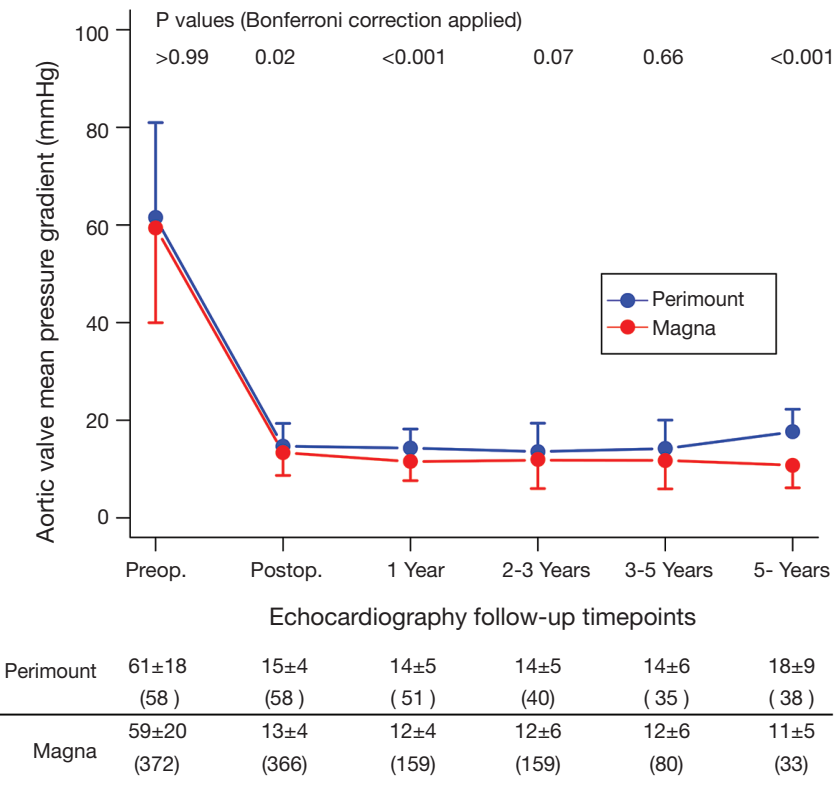

Figure 5 Serial follow-up of mean aortic valve pressure gradient $(\mathrm{mmHg})$, before inverse probability of treatment weighting (IPTW). P values were derived from generalized estimating equations with Bonferroni correction, after IPTW. Numbers of patients are shown in parentheses.

postoperative.

The Perimount valve has excellent durability, as reported by multiple studies $(3,4)$. However, the Magna valve may offer additional benefits. For example, the Magna valve's manufacturer has argued that it features improved hemodynamic performance and allows for supra-annular positioning $(5,6)$. While conventional Perimount valves are designed for both intra- and supra-annular implantation, they are more frequently implanted in the intra-annular position. Intra-annular positioning is achieved by everting sutures on the aortic annulus. In valves with a supraannular design, the sewing ring is placed on the annulus with a non-everting suture (Figure $2 C$ ). This supraannular design allows for improved haemodynamics and dynamic oversizing. In particular, one study described the hemodynamic superiority of the Magna valve in terms of its AVPG (1). Several previous reports have also concluded that the supra-annular valve has a larger measured EOA (7-9). Regardless, it is unclear whether AVPG and EOA, as measured via echocardiography, represent hemodynamic superiority (10). In particular, it has not been demonstrated whether a low AVPG or high EOA leads to improved overall survival or decreased rates of cardiac-related events
(11-15).

As with the factors mentioned above, evaluating $\mathrm{LV}$ outflow and aortic root haemodynamics is difficult due to several confounders. For instance, changes in the shape of the sinus of Valsalva after valve implantation. The relationship between the coronary orifice and the implanted valve's sewing ring is also difficult to measure precisely and each patient exhibits unique anatomy. Therefore, validating the performance of a novel valve prosthesis requires longterm clinical outcomes (e.g., overall survival).

Although our results are somewhat unclear, it is possible that the Magna valve's supra-annular design potentially worsens patients' long-term results. In addition to the original height of the coronary artery orifice, the distance between the coronary artery orifice and the valve sewing ring also varies between patients. The sewing ring can be positioned closer to the coronary artery orifice in the supraannular position rather than in the intra-annular position. Additionally, oversizing can distort the sinus of Valsalva, further reducing the distance between the coronary artery orifice and the sewing ring (Figure $2 E$ ) $(12,16)$. This problem that can be caused by supra-annular positioning becomes even more serious with a smaller sinus of Valsalva (17). A recent study showed that a smaller left coronary ostia height $(<12 \mathrm{~mm})$ is more frequently encountered in Asian populations than among Caucasians (17). Finally, left main trunk obstruction after AVR in the supra-annular position using the Magna valve has been reported previously (18). Unfortunately, due to some data being missing, we were unable to analyse the size of the sinus of Valsalva in the patients assessed in the present study.

Despite the limitations discussed above, the hemodynamic superiority of the Magna valve, as measured via AVPG, was revealed by our results. We found that Magna valve use was associated with a significantly lower AVPG during follow-up. Furthermore, it is possible that the hemodynamic benefits of this valve may become even more obvious with a larger population of patients and a longer follow-up period. The incidence of PPM with an EOA index threshold $<0.85 \mathrm{~cm}^{2} / \mathrm{m}^{2}$ (based on manufacturer-provided EOA data) was significantly higher in the Perimount group than in the Magna group. Despite this, the former exhibited improved overall and event-free survival rates $(14,19)$. We have already reported that $19-\mathrm{mm}$ Perimount valves led to favourable AVR clinical outcomes (13). The relationship between AVPG and long-term outcomes needs further investigation.

Given the retrospective nature of this study, our results 
should be interpreted with some caution. Magna valves have been used for AVR since 2005 at Samsung Medical Center. The Magna valve was the most popular replacement for its predecessor, the Perimount valve. Since March of 2017, we have used the next version of the Magna-Perimount Magna Ease valves. One study compared the Perimount, Magna, and Perimount Magna Ease valves and reported slightly improved outcomes with the first versus the other two (20). However, the study size used to obtain these results was small. We therefore cautiously conclude that there is no clear evidence that Magna valves are superior to Perimount valves in terms of their long-term clinical outcomes. Further studies with larger numbers of patients are needed, however, to validate these findings.

\section{Limitations}

While this study offers some significant benefits to the field, it is limited by its retrospective design and relatively small sample size. Also, the groups were not well balanced in terms of the number of participants included in each. Furthermore, we were unable to analyse aortic root size (sinotubular junction, sinus of Valsalva, or aortic valve annulus) or coronary artery orifice height due to missing data. Additional risk factors might have been identified had these variables been analysed. Furthermore, postoperative EOA values measured via echocardiography were unavailable. To address this limitation, we attempted to evaluate hemodynamic performance via echocardiographically measured AVPGs. A further limitation is that the Perimount and Magna valves assessed here were implanted for different periods of time with 4-5 years of overlap. Therefore, various changes in other domains of our clinical practice during the study period, many of which are unmeasurable, may have occurred. Additionally, operations performed more recently may have benefitted from improved perioperative care. However, the improvements in outcomes with Perimount valve use [1998-2005] appeared to be substantial beyond mitigation by these limitations.

To summarize, evidence should be firmly presented when we are to confirm new generation valves are better. Best implantation techniques can be an important factor to longterm clinical outcomes.

\section{Acknowledgments}

We wish to thank research assistant Joomin Hwang, who helped with data collection and management. We also wish to thank medical illustrator SeMin Oh for assistance with figure creation.

\section{Footnote}

Conflicts of Interest: The authors have no conflicts of interest to declare.

Ethical Statement: The authors are accountable for all aspects of the work in ensuring that questions related to the accuracy or integrity of any part of the work are appropriately investigated and resolved. The hospital institutional review board of Samsung Medical Center approved this study and waived any need for informed consent due to its retrospective nature (no. 2018-01-013).

\section{References}

1. Wagner IM, Eichinger WB, Bleiziffer S, et al. Influence of completely supra-annular placement of bioprostheses on exercise hemodynamics in patients with a small aortic annulus. J Thorac Cardiovasc Surg 2007;133:1234-41.

2. Akins CW, Miller DC, Turina MI, et al. Guidelines for reporting mortality and morbidity after cardiac valve interventions. Ann Thorac Surg 2008;85:1490-5.

3. Johnston DR, Soltesz EG, Vakil N, et al. Long-term durability of bioprosthetic aortic valves: implications from 12,569 implants. Ann Thorac Surg 2015;99:1239-47.

4. Guo H, Lu C, Huang H, et al. Long-Term Clinical Outcomes of the Carpentier-Edwards Perimount Pericardial Bioprosthesis in Chinese Patients with Single or Multiple Valve Replacement in Aortic, Mitral, or Tricuspid Positions. Cardiology 2017;138:97-106.

5. Dalmau MJ, Mariagonzalez-Santos J, Lopez-Rodriguez J, et al. The Carpentier-Edwards Perimount Magna aortic xenograft: a new design with an improved hemodynamic performance. Interact Cardiovasc Thorac Surg 2006;5:263-7.

6. Botzenhardt F, Eichinger WB, Guenzinger R, et al. Hemodynamic performance and incidence of patientprosthesis mismatch of the complete supraannular perimount magna bioprosthesis in the aortic position. Thorac Cardiovasc Surg 2005;53:226-30.

7. Ruzicka DJ, Hettich I, Hutter A, et al. The complete supraannular concept: in vivo hemodynamics of bovine and porcine aortic bioprostheses. Circulation 2009;120:S139-45. 
8. Gerosa G, Tarzia V, Rizzoli G, et al. Small aortic annulus: the hydrodynamic performances of 5 commercially available tissue valves. J Thorac Cardiovasc Surg 2006;131:1058-64.

9. Tayama E, Tomita Y, Takagi K, et al. New design bioprosthesis: early outcome of Carpentier-Edwards PERIMOUNT Magna in the small annular aortic position. J Artif Organs 2011;14:284-8.

10. Glaser N, Franco-Cereceda A, Sartipy U. Late survival after aortic valve replacement with the perimount versus the mosaic bioprosthesis. Ann Thorac Surg 2014;97:1314-20.

11. Carrier M, Pellerin M, Perrault LP, et al. Experience with the 19-mm Carpentier-Edwards pericardial bioprosthesis in the elderly. Ann Thorac Surg 2001;71:S249-52.

12. Cleveland JD, Bowdish ME, Eberhardt CE, et al. Evaluation of Hemodynamic Performance of Aortic Valve Bioprostheses in a Model of Oversizing. Ann Thorac Surg 2017;103:1866-76.

13. You JH, Jeong DS, Sung K, et al. Aortic Valve Replacement With Carpentier-Edwards: Hemodynamic Outcomes for the 19-mm Valve. Ann Thorac Surg 2016;101:2209-16.

14. Okamura H, Yamaguchi A, Yoshizaki T, et al. Clinical outcomes and hemodynamics of the 19-mm Perimount Magna bioprosthesis in an aortic position: comparison

Cite this article as: Chang $\mathrm{HW}$, Kim WS, Ahn JH, Carriere KC, Jeong DS, Cho YH, Sung K, Park PW. Late clinical outcomes of aortic valve replacement with Carpentier-Edwards pericardial valves. J Thorac Dis 2019;11(12):5372-5381. doi: 10.21037/ jtd.2019.11.65 with the 19-mm Medtronic Mosaic Ultra Valve. Circ J 2012;76:102-8.

15. Kume Y, Fujita T, Fukushima S, et al. Reducing ProsthesisPatient Mismatch With Edwards Magna Prosthesis for Aortic Valve Replacement. Circ J 2017;81:468-75.

16. Bottio T, Buratto E, Dal Lin C, et al. Aortic valve hydrodynamics: considerations on the absence of sinuses of Valsalva. J Heart Valve Dis 2012;21:718-23.

17. Yoon SH, Ohno Y, Araki M, et al. Comparison of aortic root anatomy and calcification distribution between Asian and Caucasian patients who underwent transcatheter aortic valve implantation. Am J Cardiol 2015;116:1566-73.

18. Nishioka N, Morimoto N, Nakagiri K, et al. A Case of Left Main Trunk (LMT) Obstruction after Aortic Valve Replacement (AVR) Using Carpentier-Edwards PERIMOUNT MAGNA. Jpn J Cardiovasc Surg 2012;41:49-52.

19. Doenst T, Amorim PA, Al-Alam N, et al. Where is the common sense in aortic valve replacement? A review of hemodynamics and sizing of stented tissue valves. J Thorac Cardiovasc Surg 2011;142:1180-7.

20. Nishioka N, Yamada A, Ujihira K, et al. Outcomes of surgical aortic valve replacement using Carpentier-Edwards PERIMOUNT bioprosthesis series in elderly patients with severe aortic valve stenosis: a retrospective cohort study. Gen Thorac Cardiovasc Surg 2016;64:728-34. 

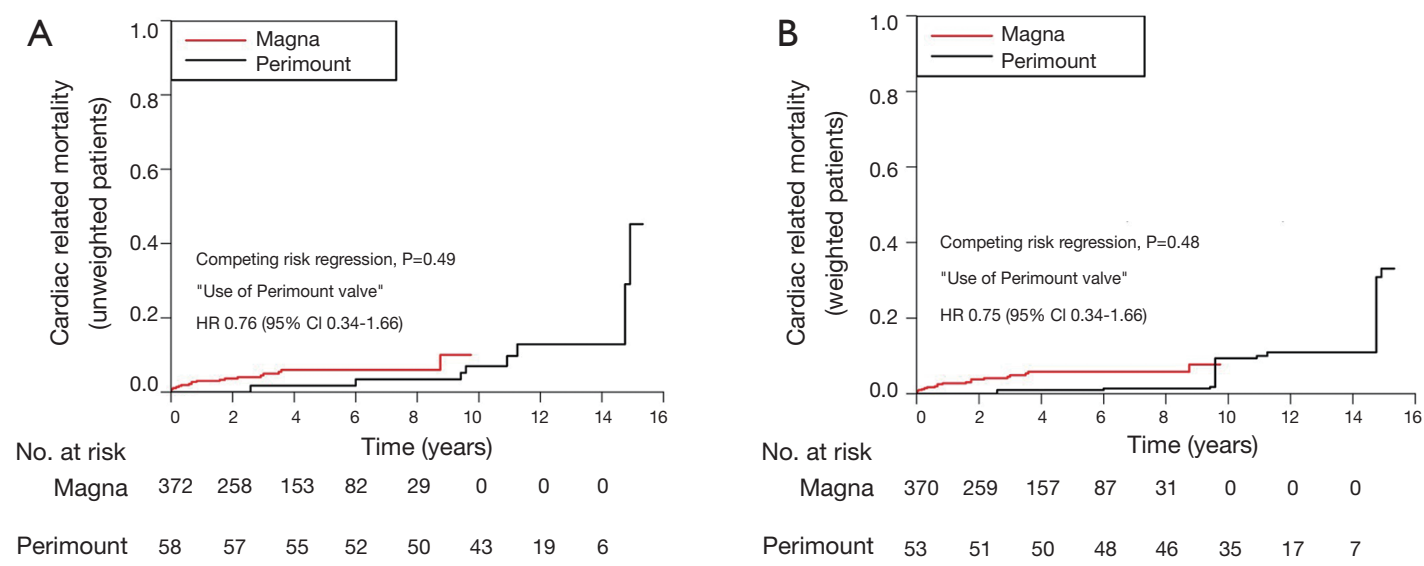

Figure S1 Cardiac related mortality. (A) Before inverse probability of treatment weighting (IPTW); (B) after IPTW. CI, confidence interval; HR, hazard ratio. 